\title{
Representações de idosos sobre a vacina da gripe
}

\author{
Representations of the elderly on the influenza vaccine
}

Susanne Pinheiro Costa e Silva ${ }^{1}$

Maria Cristina Smith Menandro ${ }^{2}$

${ }^{1}$ Colegiado de Enfermagem, Universidade Federal do Vale do São Francisco. Av. José de Sá Maniçoba s/n, Centro. Caixa Postal 252 56304-205 Petrolina PE. susanne.costa@univasf.edu.br ${ }^{2}$ Programa de Pós-Graduação em Psicologia, Universidade Federal do Espírito Santo.

\begin{abstract}
This study sought to understand the social representations of health and immunization for elderly individuals vaccinated and unvaccinated with influenza vaccine. The theoretical benchmark adopted was the Theory of Social Representations of a qualitative nature. The research was carried out with thirty elderly individuals, fifteen of whom were vaccinated against influen$z a$ and fifteen who were not. Individual interviews were conducted using a questionnaire for characterization and the Free Word Association of Test (TALP) as data collection instruments and analysis was conducted using Central Nucleus Theory. TALP data revealed differences between the representations for the elderly of the two groups. Those vaccinated considered health as being synonymous with well-being, permitting the conduct of daily activities and immunization as something that protects them from various evils. However, those who remained unvaccinated defined health as a product of divine will and immunization as something that protects, but has side effects, which discouraged them from taking it. The study indicated the importance of health education and demystification of misconceptions about vaccines, since healthy habits must be increasingly encouraged, thereby reducing the high rates of preventable morbidity and mortality.
\end{abstract}

Key words Social representations, Health, Influenza vaccine, The elderly
Resumo O presente estudo objetivou conhecer as representações sociais de saúde e imunização para idosos vacinados/não vacinados com a Influenza. Adotamos como referencial teórico a Teoria das Representações Sociais, tendo cunho qualitativo. A pesquisa foi desenvolvida com trinta idosos, sendo quinze vacinados contra a gripe e quinze não vacinados. Realizou-se entrevista individual, utilizando questionário para caracterização e Teste de Associação Livre de Palavras (TALP) como instrumentos de coleta. A análise ocorreu através da Teoria do Núcleo Central, pela ordem das evocações. Os dados revelaram diferenças entre as representações para os dois grupos: os idosos vacinados representaram a saúde como sinônimo de bemestar, que possibilita a manutenção das atividades cotidianas, e a imunização como algo que protege de diversos males; já aqueles não vacinados definiram saúde como produto da vontade divina, e a vacina como algo que protege, mas que causa variadas reações, o que os desencoraja a utilizá-la. O estudo apontou para a importância de educar em saúde e desmitificar o imaginário sobre esta como também acerca de vacinas, uma vez que hábitos saudáveis precisam ser cada dia mais estimulados, minimizando os altos índices de morbimortalidade evitáveis.

Palavras-chave Representações sociais, Saúde, Vacina Influenza, Idosos 


\section{Introdução}

Ao longo dos anos, muitas transformações concernentes à saúde da pessoa idosa têm ocorrido, motivadas pelo avanço tecnológico, que possibilitou tanto a prevenção e a recuperação de diversas doenças, como o declínio das taxas de natalidade. Com efeito, percebeu-se aumentar vertiginosamente a expectativa de vida, principalmente nos países em desenvolvimento, o que provocou a necessidade do aprimoramento de meios para a melhoria da qualidade de vida dos cidadãos com sessenta anos ou mais.

No Brasil, embora ainda distante de países desenvolvidos, a expectativa de vida em 2010 encontrou-se em torno de 73,1 anos, três a mais que o descrito em $2000^{1}$. O fenômeno do crescimento do número absoluto de idosos, bem como da sua longevidade, iniciou-se no século XX, porém acentuou-se no século subsequente. Mesmo assim, é sabido que os países em desenvolvimento, que sofrem esse processo de maneira rápida e crescente, não estão preparados para esta transição, gerando preocupação e planejamento de estratégias por parte de organizações internacionais ao redor do mundo, que buscam meios para enfrentar este processo da melhor maneira ${ }^{2}$.

No que tange à saúde coletiva, o envelhecimento populacional constitui-se como um grande desafio, especialmente pela vulnerabilidade social característica das nações que o enfrentam com maior intensidade. Conjuntamente, as transições demográfica e epidemiológica da população trazem consigo alterações significativas para a saúde, com aumento das doenças crônicas que atingem esta faixa etária e que possuem peculiaridades ${ }^{3}$.

É notável uma maior procura dos idosos por serviços de saúde bem como internações hospitalares, que geralmente são mais longas que aquelas de outras faixas etárias, traduzindo-se em maior carga de doenças, mais incapacidades e aumento do uso dos serviços de saúde ${ }^{4,5}$. Porém, sabe-se que envelhecer não é sinônimo de doença, tampouco de inaptidão para o desenvolvimento de tarefas. Atualmente, a Organização Mundial de Saúde (OMS) prioriza que o envelhecimento deve ser ativo, e não apenas saudável, como se dizia em outrora, reconhecendo que vários fatores merecem destaque já que acabam por interferir na vida cotidiana, como é o caso da cultura, oportunidades e tratamentos, entre outros ${ }^{6}$.

Isso significa dizer que a autonomia e a independência de pessoas idosas devem ser estimuladas, havendo como consequência a melhoria da qualidade de vida destes. É de vital importância ampliar a consciência sobre a saúde, hábitos de vida e o processo de envelhecimento, ao mesmo tempo fortalecendo e instrumentalizando a população idosa em suas lutas por cidadania e justiça social. Saúde e envelhecimento são indicativos de qualidade de vida, sendo que os seus significados diferem entre as pessoas ${ }^{6-8}$.

Para que o envelhecimento ativo se dê é importante que as questões de saúde também sejam consideradas. Muitas limitações decorrentes do envelhecimento são de ordens orgânicas e funcionais, que podem ser superadas ou adaptadas ao estilo de vida de cada pessoa ${ }^{7}$. Os aspectos da saúde e do viver bem entre os indivíduos idosos estão associados com os seus hábitos desde a infância até o fim da vida ${ }^{2,9}$. É necessário, então, atuar no campo do saber individual e coletivo, focando estratégias que busquem debater os aspectos que influenciam no envelhecer bem desde o nascimento.

Os estudos sobre o envelhecimento são bastante recentes, ocasionados pelo vertiginoso acontecimento nunca antes experimentado: o aumento da longevidade e da população idosa. Até bem pouco tempo pensava-se que ao envelhecer a pessoa cessava o seu desenvolvimento, adoecendo e afastando-se de suas atividades 9 . Hoje, com a superação desta crença, inúmeras ferramentas são utilizadas para retardar as problemáticas que possam acontecer com o avançar da idade, como o uso de imunobiológicos ou vacinas, fator de extrema acuidade na medicina preventiva ${ }^{2}$.

Dentre as doenças que mais acometem os idosos destaca-se a gripe, patologia evitável através de imunização. Pessoas com sessenta anos ou mais, com doenças crônicas pré-existentes, acabam sendo mais afetadas que aquelas de outras faixas etárias. Portanto, a vacinação anual de idosos é a medida mais eficaz para reduzir a morbidade e a mortalidade associadas à infecção por Influenza, e por este motivo diversas nações implementaram tal estratégia, sendo um procedimento recomendado pela OMS. Na Europa, quase todos os países aconselham a utilização da vacina contra a gripe, diversificando-se em ações para aumentar a cobertura. No entanto, a mesma é feita em clínicas particulares, o que dificulta a adesão devido ao custo ${ }^{10}$.

No Brasil, a vacina contra a gripe foi implementada no ano de 1999 no Sistema Único de Saúde com o intuito de proteger os grupos de maior risco para complicações do vírus da Influenza. Segundo o Ministério da Saúde ${ }^{11}$, houve redução da morbimortalidade, do absenteísmo no trabalho e dos gastos com tratramento de 
infecções secundárias oriundas do vírus. Sabe-se também que a imunização é a medida mais eficaz para combatê-lo, diminuindo bruscamente a incidência e a severidade da doença, embora a mesma tenha um curso benigno entre a maioria dos acometidos. Além disso, devido à falta de informação, muitos não associam as doenças subjacentes à gripe, e por este motivo muitas vezes esta não é vista como uma ameaça ${ }^{12}$.

A campanha de vacinação contra a gripe ocorre de forma prolongada em período anterior ao de maior circulação do vírus na população das diferentes regiões do Brasil. Para conferir proteção adequada, deve ser administrada a cada ano, pois sua composição também varia anualmente, em função da mutação contínua dos vírus circulantes ${ }^{11}$.

Embora sua eficácia tenha sido comprova$\mathrm{da}^{13}$, reduzindo a mortalidade por doenças respiratórias e trazendo benefícios para a saúde dos idosos, muitos deles ainda não acreditam no poder imunogênico da vacina contra influenza e a recusam. Levando-se em consideração que, embora os municípios tenham metas a cumprir, devese entender a individualidade de cada um, respeitando o direito de escolha, a educação em saúde pode atuar como forte aliada na adesão da comunidade idosa à vacina, pois muitos não aceitam por desconhecimento dos seus proveitos.

É necessário compreender também que as representações sociais a respeito do assunto influenciam inclusive na tomada de decisão dessas pessoas. Isto significa dizer que elas não estão abstraídas da realidade social, nem condenadas a reproduzí-la, mas interagem com esta. "Sua tarefa é elaborar a permanente tensão entre um mundo que já se encontra constituído e seus próprios esforços para ser um sujeito" ${ }^{14}$, como ocorre ainda pela incorporação da vacina ao cotidiano dos idosos.

Para isso, utilizam-se da objetivação e/ou da ancoragem, tornando concreto aquilo que é abstrato, familiarizando-se com o objeto e transformando-o em algo acessível na memória ${ }^{15,16}$. Na velhice, ter saúde pode estar estritamente relacionado à autonomia e independência, e por este motivo o cuidado não pode ser focalizado apenas no controle de morbidades, principalmente ao entender que a população idosa é muito peculiar? .

Internacionalmente, a vacinação configura-se como um dos indicadores utilizados para o acompanhamento da situação de saúde. Porém, nacionalmente é algo novo, cujas metas somente serão atingidas após prorrogação do tempo da campanha, indicando o conflito entre permitir-se imu- nizar ou não, interferindo nas questões de saúde e nas próprias representações sobre a temática.

Nos últimos anos, estudos em Representações Sociais têm crescido de maneira significativa no campo da saúde. Credita-se a este referencial teórico-metodológico o desenvolvimento de pesquisas que vão além do modelo mecanicista, com mais flexibilidade e fidelidade à realidade. Assim, este trabalho foi desenvolvido com o objetivo de entender como se estruturam as representações sociais de saúde e imunização para idosos, enriquecendo o debate que se dá entre os grupos de pessoas que aderem e aquelas que não se permitem vacinar.

\section{Método}

Utilizou-se como referencial teórico a Teoria das Representações Sociais ${ }^{15}$ e a Teoria do Núcleo Central $^{17}$, de abordagem qualitativa, situandose no âmbito da pesquisa social ${ }^{18}$.

A pesquisa foi realizada no município de Petrolina, localizada no Sertão Médio Pernambucano. Este município apresentou no ano de 2010 uma população de 293.962 habitantes, um crescimento de quase $30 \%$ em relação aos dados divulgados no Censo 2.000. A população domiciliada em ambiente rural foi de 74.747 habitantes ${ }^{1}$.

Sua economia baseia-se na fruticultura, com exportação de diversos produtos, tendo também a segunda produção vinícola do país. No que se refere às condições socioeconômicas, a taxa de pessoas com 60 anos ou mais sem instrução no estado de Pernambuco chega a 43,3\%, considerada muito elevada. O município possui $58 \%$ de cobertura de Equipes de Saúde, sendo 59 equipes da Estratégia Saúde da Família.

A amostra foi constituída por trinta idosos domiciliados no referido município e que atendiam aos seguintes critérios de inclusão: idade igual ou superior a 60 anos; estar cadastrado em Unidade de Saúde municipal, recebendo a visita do Agente Comunitário de Saúde (ACS); e, anuência em participar do estudo. Para a realização desta pesquisa foram selecionados quinze idosos com vacina contra a gripe atualizada e quinze que recusavam a administração do imunobiológico. A seleção foi feita com a ajuda das equipes de saúde, que indicavam os idosos que se enquadravam em tais critérios.

É importante destacar que no ano de 2010, $83,77 \%$ dos idosos da cidade de Petrolina foram imunizados, atingindo a meta mínima estabelecida pelo Ministério da Saúde, que é de $80 \%{ }^{19}$. 
A coleta de dados foi realizada por meio de entrevista, com aplicação de formulário estruturado, versando sobre questões para caracterização da amostra quanto aos dados sociodemográficos e de saúde. Utilizou-se também o Teste de Associação Livre de Palavras - TALP pelo qual o entrevistado evocava cinco termos para cada um dos estímulos Saúde / Vacina contra a Gripe, justificando as duas primeiras evocações. O tempo médio de entrevista foi de 15 minutos. Todo o conteúdo foi gravado em aparelho MP3, com posterior transcrição.

As entrevistas foram realizadas no ambiente domiciliar dos participantes. Os ACS acompanhavam o entrevistador até o domicílio dos idosos selecionados para contato inicial, no qual os objetivos do estudo eram informados, formalizando-se o convite. A depender da disponibilidade do participante, a entrevista ocorria tão logo após a conversa inicial, ou seria agendado novo encontro para aplicação das técnicas de coleta de dados. Durante a sua execução, apenas o participante e o entrevistador permaneciam no ambiente. $\mathrm{O}$ aceite de participação foi documentado mediante a assinatura do Termo de Consentimento Livre e Esclarecido, respeitando os aspectos éticos conforme a Resolução 196/96 $6^{20}$ do Conselho Nacional de Saúde. Cada participante está identificado pelo número do seu questionário, garantindo o anonimato. É válido destacar que a coleta só foi iniciada após aprovação do Comitê de Ética em Pesquisa envolvendo seres humanos da UNIVASF.

A análise foi feita no intuito de encontrar possíveis diferenças nas representações, colocandose de um lado os participantes vacinados e do outro os não vacinados. Foram identificadas setenta e cinco palavras por grupo para cada um dos termos indutores provenientes do TALP. Após a análise, tomou-se por base a frequência $(f)$ e a ordem média de evocações (OME) para identificação do Núcleo Central das Representações e sua representação na Tabela de quatro casas $^{17,21,22}$.

A interpretação dos termos evocados foi realizada pelo método da Análise de Conteúdo ${ }^{23}$. Em primeiro lugar, os dados foram organizados através da leitura flutuante, com posterior exploração do material e codificação, culminando com a formação do núcleo de compreensão dos resultados para o seu tratamento.

\section{Resultados e discussão}

\section{Caracterização dos grupos}

No intuito de distinguir os participantes do estudo quanto a questões sociodemográficas e de saúde, iniciaremos descrevendo tais indicadores. A idade média dos idosos vacinados contra a gripe foi de 70,3 anos, em sua maioria do sexo feminino $(86,6 \%)$, enquanto naqueles não vacinados a média de idades foi de 66,4 anos. Interessante notar que um estudo realizado em Porto Alegre ${ }^{12}$ identificou que os idosos mais jovens, que geralmente são mais ativos e sem grandes problemas de saúde, atendem menos ao chamado para vacinação contra a gripe, assim como pessoas do sexo masculino. Dentre os não vacinados, dois haviam recebido a vacina há alguns anos, porém não mais a aceitaram após a primeira experiência, justificando que não necessitavam de tal cuidado.

Predominaram pessoas autodeclaradas pardas em ambos os grupos, como também com baixa escolaridade - nunca estudaram ou o fizeram até o ensino fundamental, nem sempre concluído, tendo uma diferença: dentre os vacinados, uma participante havia cursado ensino superior, sendo a única que ainda trabalhava, enquanto $33 \%$ dos não vacinados estava na ativa. Como ocupação, prevaleceram trabalhadores rurais e donas-de-casa, sendo a maioria aposentados e com diagnóstico de Hipertensão e Diabetes, tendo já sido internados algumas vezes por motivos diversos, corroborando estudos que descrevem os principais usuários dos serviços de saúde $^{4,5,7}$.

\section{Representações sociais, saúde e vacinas}

As evocações provenientes do TALP foram analisadas e categorizadas de modo a considerar a dimensão individual (frequência) e a coletiva (ordem média de evocações), sendo o núcleo central identificado ${ }^{24,25}$. A classificação das categorias foi feita por estímulo indutor (saúde / vacina contra a gripe) e por grupo de participantes (idosos vacinados / não vacinados), distribuídos em tabelas. Em cada um destas tabelas, o cruzamento das linhas das médias indicadas os divide em quatro quadrantes ${ }^{26}$.

No quadrante superior esquerdo encontramse os elementos que provavelmente compõem o núcleo central das representações. No inferior direito, com elementos de menor frequência e evocação mais tardia, situam-se os elementos 
mais periféricos das representações. Os elementos dos quadrantes restantes, superior direito e inferior esquerdo, considerados evocações intermediárias, possibilitam uma interpretação menos direta, uma vez que tratam de cognições que apesar de não integrarem o núcleo central, mantêm uma relação de proximidade com este end,22 $^{2}$.

Assim, a Tabela 1 distribui os elementos que foram categorizados de acordo com as evocações feitas pelos idosos vacinados para o termo Saúde. A categoria viver bem situou-se como núcleo central para este grupo. Para os mesmos, saúde é sinônimo de alegria e bem-estar, estando o idoso tranquilo, ativo e cercado de bons sentimentos, o que o permite viver bem. É notório que os idosos vacinados compreendem a saúde como algo que continua quando a velhice chega, sendo esta uma etapa da vida como outra. Importante se faz que, como as outras fases, seja bem vivida, possibilitando a continuidade das atividades já desenvolvidas, como trabalho e lazer, feitos com alegria, prazer e bem estar.

A manutenção das atribuições remete ao idoso os aspectos positivos do envelhecer, como a independência e a funcionalidade, o que possibilita autonomia e bem estar e, apesar da idade, a pessoa deve continuar ativa como outrora ${ }^{27}$. Viver bem significa dizer que o avançar da idade não tira das pessoas a determinação de ser feliz e buscar a realização dos seus desejos, assim como tornar-se idoso não se constitui simplesmente como uma porta aberta à doença e incapacidades.

Todos os participantes foram convidados a justificarem os dois primeiros termos que evocaram. Dessa forma, a categoria viver bem, segundo aqueles que a citaram, era sinônimo de saúde porque a idade não impede ninguém de fazer nada, [...] e velho é o mundo, pois se sentem como adolescente da terceira idade, [...] e quem tem saúde tem alegria, [...] quem tem saúde tem tudo (P6). A pessoa estando com saúde está feliz, vive bem, não está com problemas (P15). Note-se que viver bem significa ter saúde na velhice, não estando a vida cotidiana prejudicada. Preocupaos a fatalidade da ausência de saúde, o que impediria a continuidade das atividades corriqueiras.

Na periferia intermediária situaram-se as categorias equipe de saúde, remédios e cuidados. Sabe-se que o público mais frequente de unidades de saúde é composto por pessoas acima de 60 anos $^{4,5}$. Desse modo, os mesmos acabam ligando saúde aos serviços prestados pelas equipes multiprofissionais, principalmente pela inserção da Estratégia Saúde da Família, que fortalece o vínculo com a população ao privilegiar ações de promoção da saúde e prevenção de doenças. A equipe é vista como corresponsável pela manutenção da saúde dos idosos, principalmente por realizar consultas periódicas de controle e continuidade de possíveis tratamentos ${ }^{28}$.

Como já dissemos, a maioria dos idosos apresentava alguma doença crônica, sendo as principais Diabetes Mellitus e Hipertensão. Talvez por este motivo muitos associaram à saúde a utilização de medicação. Sabe-se que, principalmente na terceira idade, a necessidade de intervenção medicamentosa é uma constante quando doenças crônicas se instalam, sendo grande parte dos idosos bastante dependentes de tal recurso ${ }^{7}$. O uso contínuo de medicações acaba por influenciar na qualidade de vida dos mesmos, pela obrigação do uso continuado para melhoria da saúde. De acordo com estudo realizado por Oliveira e Silva ${ }^{29}$, a escolha da clientela pelo termo remédio reforça a noção da medicalização da assistência, que acaba sendo curativista como antes, embora várias mudanças no Sistema Único de Saúde tenham realmente acontecido.

Relacionada à categoria remédios encontrase cuidados, que são fundamentais para que a saúde do idoso ocorra na prática. Por cuidados entenda-se as obrigações da pessoa para consigo mesmo, realizados de forma autônoma, como higiene, prática de exercícios físicos e alimentação balanceada. Dessa forma, a saúde é algo tangível para o idoso vacinado, mas a idade traz consigo algumas particularidades para o viver bem, como uso de medicações, acompanhamento da equipe de saúde e diversos outros cuidados que refletem na manutenção de sua saúde. Caso haja algum atropelo nesses elementos, a saúde fica comprometida e a internação acontece, o que causa bastante descontentamento no idoso por comprometer a sua rotina, retirando dele a sua autonomia.

A Tabela 2 retrata a identificação dos possíveis elementos do núcleo central do termo Saúde para os idosos não vacinados. É interessante observar que esta centralidade foi composta unicamente pelo termo Deus, como se os mesmos atribuíssem à figura divina o encargo de serem sau-

Tabela 1. Distribuição dos elementos evocados para o termo indutor saúde pelos idosos vacinados.

\begin{tabular}{llrlllll}
\hline & \multicolumn{3}{c}{ O.M.E. $<\mathbf{3 , 2 2}$} & & \multicolumn{2}{c}{ O.M.E. $\geq \mathbf{3 , 2 2}$} \\
\cline { 2 - 3 } Freq. $\geq 15$ & Viver bem & 27 & 2,88 & & Cuidados 18 & 3,66 \\
Freq. $<15$ & Equipe de saúde & 7 & 2,42 & & Internação 11 & 4,27 \\
& Remédios & 12 & 2,91 & & & & \\
\end{tabular}




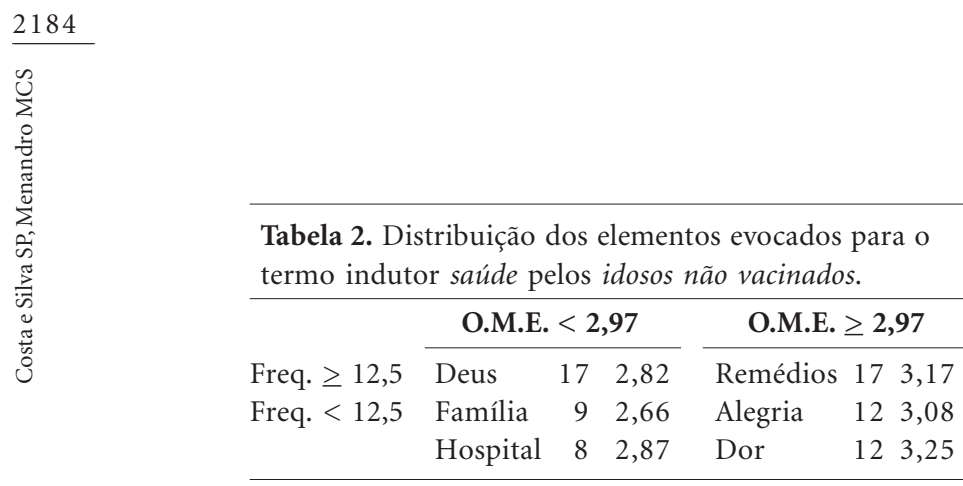

dáveis, sendo a saúde algo mítico e guiado pela fé. É possível que essa representação ancore-se no ideário da saúde como merecimento ou prêmio, e sua ausência poderia ser um castigo dado por Deus, o protetor da vida. Ressalta-se aqui a importância de valorizar junto aos idosos práticas que apreciem a percepção deles para o autogerenciamento do cuidado próprio com a saúde, favorecendo sua expectativa quanto ao futuro ${ }^{30}$.

Os participantes não vacinados justificaram sua representação de saúde como ancorada na figura de Deus porque é de um ser supremo que as coisas surgem, inclusive o fato de ter doenças ou não. Segundo os mesmos, todos tem que ter fé na providência, porque só existe Deus (P22), e é pela fé que se vive mais e que vamos viver mais e não ficando doente, mas sim com saúde como ago$r a$ andando, enxergando e confiando que Deus dá saúde até o fim da vida (P20), já que todos temos um ser superior que dá tudo (P24).

As categorias família, hospital e remédios situaram-se na periferia intermediária. Um estudo realizado por Wachelke et al. ${ }^{8}$ demonstrou que a proximidade familiar é marcante quando se envelhece, especialmente quando este é marcado por desgaste e mudanças físicas, ou mesmo por preocupações com a saúde. Assim, é interessante percebermos que este sentido é complementado pelo desenho do hospital na mente dos participantes, principalmente porque se sabe que o número de internações hospitalares tem aumentado vertiginosamente, mesmo com todas as atenções disponíveis atualmente ${ }^{31}$.

Para este grupo, saúde, além de algo orientado por um ser superior, pauta-se nos problemas que podem ocorrer com a terceira idade e que são minimizados ou mesmo cuidados com a ajuda dos familiares próximos, como se houvesse uma maior união da unidade familiar quando se torna idoso e espera-se ficar com menos saúde. Mesmo que estes idosos sejam mais ativos que os vacinados, a saúde parece algo que os inquieta, não parecendo ser tratada como algo decorrente de uma preocupação ao longo da vida, mas sim da vontade de Deus. A saúde como algo po- sitivo no envelhecimento situou-se apenas na periferia mais distante, com a categoria alegria, que vem acompanhada de dor. Como no estudo de Vilarino et al. ${ }^{12}$, os idosos não vacinados se sentem mais saudáveis e ativos, porém são também mais sedentários, o que justifica o aparecimento da categoria dor.

No Tabela 3 encontramos as representações das pessoas vacinadas quanto ao termo indutor Vacina contra a gripe. Note-se que para eles, o termo remete à prevenção como núcleo central. É através da vacina Influenza, oferecida na campanha anual para os idosos brasileiros, que muitas doenças do aparelho respiratório são evitadas, sendo esta um cuidado que promove segurança. Ela surge como estratégia utilizada pelos idosos para manter a saúde e o bem-estar, na tentativa de garantir um envelhecimento bem sucedido, como referem Silva et al..$^{32}$.

A associação do estímulo vacina à prevenção provavelmente deu-se porque ela [a vacina] é favorável à saúde, cortando muitos malefícios da doença, evitando muita coisa, [...] e não pode tomar depois de adoecer, tem que tomar antes (P7), o que mostra a importância de evitar a instalação de patologias pelo uso da mesma. A vacina aparece como um elemento que ativa as defesas imunológicas, [...] e quem toma vacina sabe que adquire defesas (P4). Aqueles que aderem à campanha anual mostraram conhecer a importância de prevenir doenças através do uso do imunobiológico, fato importante para que os mesmos não desistam da ação nas campanhas subsequentes, embora o grau de conhecimento acerca do tema não tenha sido verificado.

Vários estudos ${ }^{10,12,33,34}$ relatam a eficácia da vacina contra Influenza para idosos. Existe no meio científico comprovação de que aqueles que participam das campanhas geralmente não apresentam episódios de gripe, estando menos vulneráveis ao adoecimento ${ }^{33}$. Também é importante considerar a diminuição do número de internações por Infecção Respiratória Aguda em idosos após o início do programa que instituiu o imuno ${ }^{12,34}$, o que nos leva a crer que a prevenção está sendo efetiva e sentida por aqueles que buscam vacinar-se. Para eles, ocorre prevenção não só contra a gripe, mas também de infecções, internações hospitalares, incapacidades e muitas outras coisas que tornam o idoso vulnerável quando doente, sendo esta precaução um caminho para manter autonomia e saúde, termo presente nas evocações intermediárias.

Embora os benefícios ocasionados pela vacina sejam levados em consideração, os partici- 
pantes vacinados referiram que a mesma causa dor e desconforto, mas que apesar disso é importante vacinar-se porque várias doenças já foram erradicadas do nosso território como benefício desta ação. Tudo isto sugere que os idosos do grupo de vacinados representam a vacina de forma positiva, mesmo com o mal estar que pode vir a ocasionar.

A apresentação dos elementos evocados pelos participantes não vacinados para o estímulo Vacina contra a gripe pode ser conferida na Tabela 4 . O núcleo central compôs-se das categorias reação e prevenção. Essa centralidade nos revela a dicotomia existente entre os idosos que não aderiam à imunização, pois em primeiro lugar surgiu o ideário de que a vacina causa reações, muito embora demonstrassem conhecer a finalidade da mesma, que é prevenir doenças. $\mathrm{O}$ imuno, então, é visto como algo capaz de alcançar um objetivo, porém devido às consequências que podem decorrer dele, opta-se por não utilizá-lo.

Os termos que compõem este núcleo central foram justificados pelos participantes como fatores que explicam a não vacinação devido ao medo da reação, que causa desgosto (P16), e às vezes a pessoa tem outro problema e a vacina prejudica, acontecem outras coisas, as reações (P20), tem mal estar quando toma, sente aquela coisa ruim (P23). E no intuinto de evitar que tais reações aconteçam, mesmo sabendo que a saúde é melhor que a [possibilidade de ter] doença (P25), e que é a obrigação tomar, porque prevenir é o melhor remédio (P22), preferiram não prevenir-se contra a gripe e outras patologias associadas.

Alguns estudos ${ }^{33,35}$ citam que o principal fator que impede idosos de participar das campanhas de vacinação é a ocorrência de reações adversas, já que muitos deles afirmam ter sentido dores musculares e adquirido gripe como evento associado à vacina. Faz-se necessário entender que qualquer sinal ou sintoma grave ou inesperado que ocorra após a vacinação pode ser um evento adverso. Estes estão associados diretamente à vacina e seus efeitos no organismo, como também por erro programático decorrente do preparo, manejo ou administração inadequados da vacina. Pode, inclusive, haver coincidência na associação temporal, surgindo sintomas que não decorrem da vacina ${ }^{36}$.

Outro aspecto que necessita de atenção é que a vacina influenza, quando muito, apresenta reações benignas e locais, sendo responsável por pequeno número de notificações ${ }^{37}$. Dessa forma, é imprescindível que o profissional oriente o usuário para a possibilidade de reações e quais se-
Tabela 3. Distribuição dos elementos evocados para o termo indutor vacina contra a gripe pelos idosos vacinados.

\begin{tabular}{lllllll}
\hline & \multicolumn{2}{c}{ O.M.E. $<2,94$} & & \multicolumn{2}{c}{ O.M.E. $\geq \mathbf{2 , 9 4}$} \\
\cline { 2 - 3 } Freq. $\geq 18,75$ & Prevenção & 19 & 2,78 & & Doenças superadas 23 & 3,65 \\
Freq. $<18,75$ & Dor & 16 & 2,87 & & & \\
\cline { 5 - 6 } & Saúde & 17 & 2,47 & & \\
\hline
\end{tabular}

Tabela 4. Distribuição dos elementos evocados para o termo indutor vacina contra a gripe pelos idosos não vacinados.

\begin{tabular}{llrrrrrr}
\hline & \multicolumn{3}{c}{ O.M.E. $<\mathbf{3 , 0 1}$} & & \multicolumn{3}{c}{ O.M.E. $\geq \mathbf{3 , 0 1}$} \\
\cline { 2 - 3 } \cline { 6 - 7 } Freq. $\geq 15$ & Reação & 21 & 2,95 & & Medo & 18 & 3,2 \\
Freq. $<15$ & Prevenção & 20 & 2,8 & & Morte & 10 & 3,1 \\
& Outros & 3 & 6 & & & & \\
\hline
\end{tabular}

jam estas, evitando-se distorções que afastem a comunidade da vacinação sistemática e diminua a cobertura vacinal ${ }^{38}$. Assim, o medo da morte, das reações e outros que possam surgir, que apareceram nas periferias das representações, pode ser trabalhado através da educação em saúde com toda a comunidade, especialmente aquela que se encaixa nas especificações para receber a vacina influenza.

A análise dos elementos de representação de saúde e gripe para o grupo de idosos não vacinados nos auxilia a entender o conjunto de elementos presentes na representação de vacina contra a gripe desse mesmo grupo, e quais deles parecem direcionar as práticas dos mesmos. Como o elemento central para saúde é Deus, esta é representada como algo que é concedido ou não por um ser supremo por motivos que nem sempre estão necessariamente relacionados aos comportamentos preventivos. Assim, na representação de vacina contra a gripe, apesar de presença do elemento Prevenção, parecem prevalecer a reação e o medo da morte, o que os permite não aderirem a campanhas de imunização para tal.

\section{Considerações finais}

De maneira geral, os idosos que fizeram parte deste estudo foram bastante heterogêneos quando comparados os dois grupos. Aqueles que aderem à vacinação contra a gripe parecem ser mais velhos, em sua maioria mulheres e não mais tra- 
balham. Já os não vacinados são mais jovens, com maior proporção de homens que o grupo anterior e com mais pessoas inseridas no mercado de trabalho. Essas características dos grupos permitem inferir que os idosos não vacinados sentem-se mais saudáveis que os vacinados, o que de certa forma influencia suas representações e suas práticas sobre saúde e vacina.

Para os idosos que se vacinavam nas campanhas anuais, saúde teve como núcleo central a categoria viver bem, representada como algo que a velhice não destrói caso os hábitos de vida de cada um contribuam para tal. Atividades de lazer, independência e autonomia ligam-se a esta concepção, contrariando os pressupostos de que a senescência traz consigo a ideia de inutilidade que por muitos anos difundiu-se. Para que a saúde se dê, conta-se com o apoio de medicações, profissionais de saúde e alguns cuidados como alimentação, exercícios e muitos outros, mantendo a qualidade de vida. A vacina contra a gripe representou para eles a prevenção de muitas doenças que atingem um grande contingente de idosos, auxiliando na manutenção da saúde.

Entretanto, os idosos que não aderiam às campanhas parecem isentar-se da responsabili- dade de atitudes que promovam saúde, representando-a como algo dado por Deus, que a distribui segundo a fé de cada um. A família figurou como importante no apoio à saúde, estando esta naturalmente comprometida quando da chegada da terceira idade. Embora declarem entender a vacina como propulsora da prevenção de doenças, as reações parecem ter mais força como elemento na sua representação, e aliado ao Medo de que elas ocorram, optam por evitar o seu uso.

Diante da realidade encontrada sugere-se que estratégias utilizadas para a captação de idosos com o intuito da adesão à vacina sejam intensificadas, discutindo modos de viver bem e a importância da prevenção em saúde, desmitificando o ideário de que a vacina antigripal provoque efeitos colaterais graves. $\mathrm{O}$ estímulo aos cuidados em saúde deve ocorrer desde o início da vida, contribuindo para a formação de consciência crítica e auxiliando na velhice saudável. Recomenda-se também que o debate sobre estas questões seja ampliado, motivado principalmente pelo grande contingente de idosos que já se tem e que tende a aumentar ainda mais. Discutir o assunto significa preocupar-se com a qualidade de vida das pessoas com sessenta anos e mais.

\section{Colaboradores}

SP Costa e Silva foi autora da concepção teórica, coleta e análise dos dados, bem como da redação do texto; MCS Menandro orientou todas as etapas do trabalho e colaborou na redação final.

\section{Agradecimentos}

À Fundação de Amparo à Ciência e Tecnologia do Estado de Pernambuco - FACEPE, pelo apoio e custeio de bolsa de doutorado. Aos respondentes, pela participação voluntária e contribuição para a ciência. 


\section{Referências}

1. IBGE. Censo Demográfico 2010. [página na Internet]. [acessado 2011 abr 10]. Disponível em: http:/ /www.censo2010.ibge.gov.br/

2. Stuckelberger A, Tellier S, Vikat A. Succès et défi du vieillissement global de la population: un plan d'action unique entre Nations Unies, scientifiques et organisations non gouvernementales. Rev Med Suisse 2009; 5:S63-S67.

3. Dallepiane LB. Envelhecimento Humano: Campo de saberes e práticas em Saúde Coletiva. Ijuí: Unijuí; 2009.

4. Veras R. Envelhecimento populacional contemporâneo: demandas, desafios e inovações. Rev Saude Publica 2009; 43(3):548-554.

5. Del Ducca GF, Thumé E, Hallal PC. Prevalência e fatores associados ao cuidado domiciliar a idosos. Rev Saúde Publica 2011; 45(1):113-120.

6. Keinert TMM, Rosa TEC. Direitos Humanos, envelhecimento ativo e saúde da pessoa idosa: marco legal e institucional. BIS, Bol Inst Saúde 2009; 47:4-8.

7. Martins JJ, Schneider DG, Coelho FL, Nascimento ERP, Albuquerque GL, Erdmann AL, Gama FO. Avaliação da qualidade de vida de idosos que recebem cuidados domiciliares. Acta paul. enferm. 2009; 22(3):265-271.

8. Wachelke JFR, Camargo BV, Hazan JV, Soares DR, Oliveira LTP, Reynaud PD. Princípios organizadores da representação social do envelhecimento: dados coletados via internet. Estud Psicol (Natal) 2008; 13(2):107-116.

9. Martins CRM, Camargo BV, Biasus F. Representações sociais do idoso e da velhice de diferentes faixas etárias. Univ Psychol 2009; 8(3):831-847.

10. Kramarz P, Ciancio B, Nicoll A. Seasonal and pandemic influenza vaccines for the elderly and other risk groups: A review of available data. Arch Med Wewnêtrznej 2009; 119(10):654-658.

11. Brasil. Ministério da Saúde (MS). Guia de Vigilância Epidemiológica. 7a Edição. Brasília: MS; 2009.

12. Vilarino MAM, Lopes MJM, Bueno ALM, Brito MRV. Idosos vacinados e não vacinados contra a influenza: morbidade relatada e aspectos sociodemográficos, Porto Alegre (RS, Brasil), 2004. Cien Saude Colet 2010; 15(6):2879-2886

13. Francisco PMSB, Donalisio MRC, Lattorre MRDO. Impacto da vacinação contra influenza na mortalidade por doenças respiratórias em idosos. Rev Saude Publica 2005; 39(1):75-81.

14. Jovchelovitch S. Vivendo a vida com os outros: intersubjetividade, espaço público e representações sociais. In: Guareschi PA, Jovchelovitch S, organizadores. Textos em representações sociais. $8^{\text {a }}$ Edição. Petrópolis: Vozes; 1994. p. 78

15. Moscovici S. Representações Sociais: investigações em psicologia social. Petrópolis: Vozes; 2003.

16. Trindade ZA, Santos MFS, Almeida AMO. Ancoragem: notas sobre consensos e dissensos. In: Almeida AMO, Santos MFS, Trindade ZA, organizadores. Teoria das Representações Sociais: 50 anos. Brasília: Technopolitik; 2011.
17. Abric JC. L’organization interne des représentations sociales: système central et système périphérique. In: Guimelli C, organizador. Struture et transformations des représentations sociales. Lausanne: Delachaux et Nestlié; 1994. p. 73-83.

18. Minayo MCS. Avaliação por triangulação de métodos. Rio de Janeiro: Fiocruz; 2005.

19. Brasil. Sistema de Informação do Programa Nacional de Imunização. Campanha Nacional de Vacinação contra Gripe 2010. [página na Internet]. [acessado 2013 jun 13]. Disponível em: http://pni.datasus. gov.br/Consulta_Gripe_Mun_10.asp?UF=26\&faixas $=$ total

20. Brasil. Ministério da Saúde (MS). Conselho Nacional de Saúde. Resolução no 196 de 10 de outubro de 1996. Diretrizes e Normas Regulamentadoras de Pesquisas Envolvendo Seres Humanos. Diário Oficial da União 1996; 16 out.

21. Sá CP. Núcleo Central das Representações Sociais. 2a Edição. Petrópolis: Vozes; 2002.

22. Oliveira DC, Marques SC, Gomes AMT, Teixeira MCTV. Análise das Evocações Livres: uma Técnica de Análise Estrutural das Representações Sociais. In: Oliveira DC, Marques SC, Gomes AMT, Teixeira MCTV, Moreira ASP, organizadores. Perspectivas Teórico-Metodológicas em Representações Sociais. João Pessoa: UFPB; 2005. p. 573-603.

23. Bardin L. Análise de conteúdo. 3a Edição. Lisboa: Ed. 70; 2004

24. Abric JC. A abordagem estrutural das representacões sociais. In: Abric JC, Moreira ASP, Oliveira DC. Estudos Interdisciplinares de Representação Social. Goiânia: AB, 2000. p. 27-38.

25. Vergès P. Approche du noyau central: propriétés quantitatives et structurales. In: Vergès PC. Structures et transformations des représentations sociales. Lausanne: Delachaux et Niestlé; 1994. p. 233-249.

26. Bergan C, Bursztyn I, Santos COM, Tura LFR. Humanização: representações sociais do hospital pediátrico. Rev. Gaúcha Enferm 2009; 30(4):656-661.

27. Ferreira OGL, Maciel SC, Silva AO, Sá RCN, Moreira MASP. Significados atribuídos ao envelhecimento: idoso, velho e idoso ativo. Psico-USF 2010; 15(3):357-364

28. Martins PC. De quem é o SUS? Sobre as representações sociais dos usuários do Programa Saúde da Família. Cien Saude Colet 2011; 16(3):1933-1942.

29. Oliveira DC, Silva LL. O que pensam os usuários sobre a saúde: representação social do Sistema Único de Saúde. Rev Enferm UERJ 2010; 18(1):14-18.

30. Clarck DO, Frankel RM, Morgan DL, Ricketts G, Bair MJ, Nyland KA, Callahan CM. The meaning and significance of self-manegement among socioeconomically vulnerable older adults. J. Gerontol B Psychol Sci Soc Sci 2008; 63B(5):5312-5318.

31. Virtuoso JF, Balbé GP, Mazo GZ, Pereira MGS, Santos FS. Morbidade e mortalidade da população idosa de Florianópolis: um estudo comparativo entre homens e mulheres. Rev Bras Geriatr Gerontol 2010; 13(2):215-223. 
32. Silva HS, Lima AMM, Galhardoni R. Envelhecimento bem-sucedido e vulnerabilidade em saúde: aproximações e perspectivas. Interface (Botucatu) 2010; 14(35):867-877.

33. Santos ZMG, Oliveira MLC. Avaliação dos conhecimentos, atitudes e práticas de idosos sobre a vacina contra a Influenza, na UBS, Taguatinga, DF, 2009. Epidmiol Serv Saúde 2010; 19(3):205-216.

34. Góis ALB, Veras RP. Informações sobre a morbidade hospitalar em idosos nas internações do Sistema Único de Saúde do Brasil. Cien Saude Colet 2010; 15(6):2859-2869.

35. Reis PO, Nozawa MR. Análise do programa de vacinação de idosos de Campinas, SP. Cien Saude Colet 2007; 12(5):1353-1361.

36. Waldman EA, Luhm KR, Monteiro SAMG, Freitas FRM. Vigilância de eventos adversos pós-vacinação e segurança de programas de imunização. Rev Saude Publica 2011; 45(1):173-184.

37. Piacentini S, Moreno LC. Eventos adversos pósvacinais no município de Campo Grande (MS, Brasil). Cien Saude Colet 2011; 16(2):531-536.

38. Luna GLM, Vieira LJES, Souza PF, Lira SVG, Moreira DP, Pereira AS. Aspectos relacionados à administração e conservação de vacinas em centros de saúde no Nordeste do Brasil. Cien Saude Colet 2011; 16(2):513-521.

Artigo apresentado em 08/06/2012

Aprovado em 17/07/2012

Versão final apresentada em 01/08/2012 